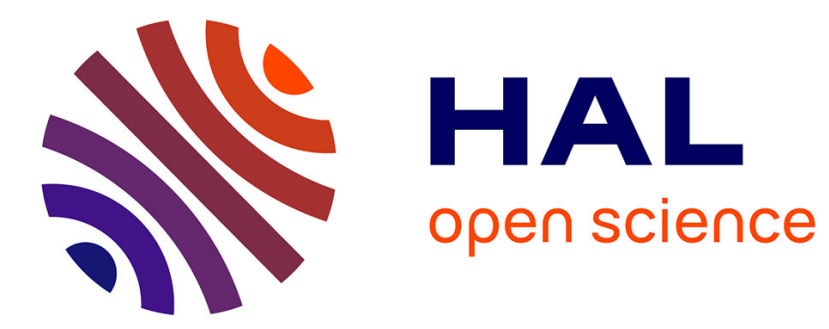

\title{
La transmission croisée dans la résistance aux antibiotiques : son contrôle dans les hôpitaux français
}

Vincent Jarlier

\section{To cite this version:}

Vincent Jarlier. La transmission croisée dans la résistance aux antibiotiques : son contrôle dans les hôpitaux français. Bulletin de l'Académie Nationale de Médecine, 2019, 203 (3-4), pp.170-178. 10.1016/j.banm.2019.04.001 . hal-02289430

\section{HAL Id: hal-02289430 https://hal.sorbonne-universite.fr/hal-02289430}

Submitted on 16 Sep 2019

HAL is a multi-disciplinary open access archive for the deposit and dissemination of scientific research documents, whether they are published or not. The documents may come from teaching and research institutions in France or abroad, or from public or private research centers.
L'archive ouverte pluridisciplinaire HAL, est destinée au dépôt et à la diffusion de documents scientifiques de niveau recherche, publiés ou non, émanant des établissements d'enseignement et de recherche français ou étrangers, des laboratoires publics ou privés. 


\title{
La transmission croisée dans la résistance aux antibiotiques; son contrôle
}

\author{
dans les hôpitaux français.
}

\section{Cross transmission in antibiotic resistance ; control in French hospitals}

\begin{abstract}
Vincent Jarlier, Sorbonne Universités, Faculté de Médecine Pierre et Marie Curie, Centre d'Immunologie et des Maladies Infectieuses, UMR 1135 ; Laboratoire de BactériologieHygiène Groupe hospitalier Pitié-Salpêtrière-Charles Foix, Paris ; Délégué aux infections nosocomiales, Direction de l'Assistance publique - Hôpitaux de Paris.
\end{abstract}

Adresse : laboratoire de Bactériologie-Hygiène, hôpital Pitié-Salpêtrière, 47-83 bd de l'Hôpital - 75651 Paris cedex 13

E-mail : vincent.jarlier@aphp.fr

L'auteur déclare n'avoir aucun lien d'intérêt avec le contenu de cet article.

\section{Résumé}

La résistance acquise aux antibiotiques est la conséquence directe de deux causes synergiques: l'utilisation massive d'antibiotiques qui entraîne la sélection des bactéries les plus résistantes (évolution darwinienne), et la dissémination des bactéries résistantes ainsi sélectionnées, par transmission au sein des populations humaines et animales ( transmission croisée »), et via l'environnement. Les programmes de lutte contre la résistance bactérienne doivent donc associer des mesures visant à contrôler la transmission croisée aux mesures visant à diminuer la consommation des antibiotiques. Des programmes de contrôle de la dissémination des bactéries multirésistantes ont été progressivement mis en place dans les hôpitaux de l'Assistance Publique - Hôpitaux de Paris (APHP) depuis 1993 puis dans l'ensemble des hôpitaux français depuis 1999. Ces programmes ont permis d'obtenir une forte diminution des taux de staphylocoques multirésistants et de garder très bas les taux de bactéries hautement résistantes émergentes : entérobactéries résistantes aux carbapénèmes ( $\beta$ lactamines les plus récentes) par production de carbapénèmases et entérocoques résistants aux glycopeptides. Ces résultats ont été obtenus alors même que la consommation d'antibiotiques n'avait pas diminué de façon notable dans nos hôpitaux durant la période considérée montrant l'impact positif des mesures d'hygiène.

\section{Summary}

The acquired resistance to antibiotics is the direct consequence of two synergic causes: the massive use of antibiotics which pulls the selection of the most resistant bacteria (Darwinian evolution), and the spread of the resistant bacteria so selected, by transmission within human and animal populations ("cross transmission"), and via the environment. Thus the programs to control bacterial resistance should combine measures for limiting cross transmission with measures for decreasing antibiotic consumption. Programs to control multiresistant bacteria dissemination were gradually implemented in the hospitals of l'Assistance Publique Hôpitaux de Paris (APHP) since 1993 and in all French hospitals since 1999. These programs have resulted in a dramatic decrease in multi-drug resistant staphylococci and in keeping very low the rates of highly resistant emergent bacteria: enterobacteria resistant to carbapenems (most recent lactams) by the production of carbapenemases and glycopeptide resistant 
enterococci. These results have been achieved even though the consumption of antibiotics has not significantly decreased in our hospitals during the period considered, demonstrating the positive impact of measures of hygiene.

\section{Mots-clef}

Résistance acquise aux antibiotiques, transmission croisée, programmes de contrôle, contrôle des staphylocoques multirésistants, contrôle des entérobactéries et entérocoques multirésistants

\section{Keywords}

Acquired antibiotic resistance, spread by cross transmission, control programs, control of multiresistant staphylococci, control of multiresistant enterobacteria and enterococci.

\section{Introduction}

Dès le début de l'utilisation en médecine des deux premiers antibiotiques, sulfamides en 1935 et pénicilline en 1942, il est apparu que certaines espèces bactériennes leurs étaient intrinsèquement résistantes (résistance dite «naturelle ») et que des souches d'espèces naturellement sensibles ne l'étaient plus (résistance dite «acquise »). La résistance naturelle fait partie des caractéristiques génétiques propres à certaines espèces, par exemple tous les bacilles de la tuberculose résistent à la plupart des antibiotiques classiques (pénicillines, sulfamides, chloramphénicol, érythromycine...). La résistance acquise résulte de la capacité des bactéries de s'adapter à la pression exercée par les antibiotiques en modifiant leur génome par mutation chromosomique ou acquisition de gènes «étrangers » à partir d'autres bactéries (transfert horizontal de gènes de résistance).

La résistance acquise aux antibiotiques est la conséquence délétère directe de deux causes synergiques liées à l'activité humaine :.(a) l'utilisation massive d'antibiotiques en santé humaine et animale, qui entraîne la sélection des bactéries les plus résistantes (évolution darwinienne), et (b) la dissémination des bactéries résistantes ainsi sélectionnées, directement par transmission au sein des populations humaines et animales («transmission croisée »), et indirectement via l'environnement.

Parce que le développement de l'antibiorésistance met en péril la sécurité sanitaire des populations, les plus hautes autorités politiques, institutionnelles et scientifiques de la planète se sont saisies de ce sujet. En 2015, l'OMS a lancé un plan d'action mondial pour combattre la résistance aux agents antimicrobiens (1). L'assemblée générale des Nations Unies, qui ne se saisit que très rarement de sujets de santé, a proposé en septembre 2016 (2) une approche coordonnée pour s'attaquer aux causes de la résistance aux agents antimicrobiens au sens large (antibiotiques, antiviraux, antiparasitaires). L'Union Européenne a aussi intégré ces dernières années à sa politique communautaire le risque sanitaire que représente l'antibiorésistance. Un $1^{\text {er }}$ plan quinquennal d'action contre la résistance microbienne a été initié en 2011 (3), suivi d'un 2ème plan lancé en juin 2017 (4), englobant les composantes nieau humaines et animales selon le concept « One Health».

En 2016, un rapport rédigé à la demande de la Ministre des Affaires sociales et de la Santé Marisol Touraine par le Docteur Jean Carlet à partir du travail collégial de nombreux experts (rapport «Carlet») a listé une série de recommandations scientifiques et techniques pour combattre l'antibiorésistance et qui en ciblent les deux causes synergiques listées plus haut, la pression de sélection par les antibiotiques et la dissémination des bactéries résistantes (5). 


\section{Multirésistance et transmission croisée}

Les mutations et gènes de résistances étrangers peuvent s'accumuler dans une même bactérie par cycles successifs de pressions de sélection et de transmissions croisées, aboutissant à la multirésistance,entraînant de sérieuses difficultés thérapeutiques pouvant aboutir à des impasses. La transmission croisée joue un rôle central dans le développement de la multirésistance aux antibiotiques, comme cela a été largement décrit dans les maladies contagieuses (tuberculose, gonococcies...), mais aussi dans les infections provoquées par les bactéries opportunistes (staphylocoques, entérobactéries...). Il faut remarquer que les bactéries responsables d'infections opportunistes qui ne font pas l'objet de transmission croisée (mycobactéries non tuberculeuses, listéria, bacillus...) sont peu ou pas concernées par le phénomène de résistance acquise. La complexité des multiples événements génétiques (imbrication de mutations chromosomiques et/ou d'acquisition d'éléments mobiles composites : plasmides, transposons, intégrons ...) qui ont conduit à la multirésistance exclut la possibilité de créer de novo ces types de bactéries chez chaque nouveau patient. C'est le cas des staphylocoques dorés (Staphylococcus aureus résistant à la méticilline ou SARM). C'est le cas aussi des entérobactéries (résistance aux nouvelles céphalosporines par production de $\beta$-lactamases à spectre étendu [EBLSE] ou aux carbapénèmes, $\beta$-lactamines les plus récentes, par production de carbapénèmases [EPC]) et des entérocoques (résistance aux glycopeptides [ERG]). Ce qui assure le succès épidémiologique de ces «monstres génétiques » une fois obtenus est leur transmission au sein des populations humaines ou animales, directement ou par l'intermédiaire de réservoirs intermédiaires (environnement ...), même si, bien sûr, pression antibiotique joue un rôle clef dans le maintien de ces bactéries dans les hôtes contaminés. Les bactéries multirésistantes (BMR) se propagent ainsi dans les établissements de soins mais aussi la population générale. Un travail basé sur les statistiques disponibles pour l'année 2012 a permis d'évaluer à 158,000 le nombre d'infections à bactéries multirésistantes survenues cette année-là en France, aboutissant à environ 13.000 décès (6), chiffre 3 fois supérieur à celui des décès par accidents de la route !

La France a engagé depuis de nombreuses années des actions visant à réduire la consommation des antibiotiques pour diminuer la pression de sélection. La campagne des années 2000 ( «les antibiotiques c'est pas automatique »), puis les Plans «EcoAntibio » ont permis de réduire partiellement et transitoirement la consommation en médecine humaines mais ont été plus efficaces en médecine vétérinaire. Ces actions ne sont pas développées dans le présent chapitre qui se concentre sur les programmes de contrôle de la diffusion épidémique des bactéries multirésistantes (BMR).

Contrôler la propagation des BMR les plus fréquentes (SARM, EBLSE), et celle des plus résistantes qui ont récemment émergées en France (EPC, ERG) et contre lesquelles les possibilités thérapeutiques sont extrêmement réduites, est un défi pour les établissements de soins. L'Assistance Publique - Hôpitaux de Paris (APHP), plus large institution publique hospitalière de France, a été pionnière dans la mise en place de programmes de surveillance et de contrôle des BMR.

\section{Programmes institutionnels de contrôle des bactéries multirésistantes à l'Assistance Publique - Hôpitaux de Paris}

L'APHP regroupe 38 hôpitaux universitaires (22 de court séjour et 16 de réadaptation / soins de longue durée), totalise 21000 lits (10\% des lits des hôpitaux publics de France) et desservant 12 millions d'habitants et admet environ 1 million de patients hospitalisés par an. Les Equipes Opérationnelles d'Hygiène hospitalière $(\mathrm{EOH})$ présentes dans chaque hôpital sont chargées de la prévention et de la surveillance des infections associées aux soins. Les 
décisions stratégiques communes à l'ensemble de l'institution sont coordonnées par une équipe multidisciplinaire centrale d'hygiène hospitalière (infectiologue, bactériologiste, épidémiologiste et cadre infirmier). Les programmes institutionnels visant à contrôler la diffusion des BMR dans les hôpitaux de l'APHP ont été initié en 1993 et ont successivement inclus différentes mesures: (a) précautions contact contre les SARM, promotion de l'utilisation des solutions hydro-alcooliques pour l'hygiène des mains, (b) mesures renforcées contre les bactéries émergentes les plus résistantes (EPC, ERG), (c) politique de gestion des excréta. Ces «programmes BMR », en grande partie inspirés d'expériences ayant démontré leur efficacité à l'étranger (7-11) ont été déployés par étapes par les $\mathrm{EOH}$ locales et ont été soutenues par un engagement fort des administrations centrales et locales.

La 1ère étape, en 1993, a mis en place des mesures pour lutter contre la transmission croisée des SARM dont l'incidence était alors plus élevée en France que dans les autres pays européens. Les mesures «SARM» incluaient l'identification des patients infectés, les précautions contact, la formation et la rétro information régulière des équipes sur les résultats obtenus.

La 2ème étape a été une campagne lancée en 2001-2002 pour promouvoir l'utilisation des solutions hydro-alcooliques pour l'hygiène des mains (SHA). Du matériel pédagogique spécifique a été mis à la disposition des $\mathrm{EOH}$ et une lettre du Directeur Général a demandé à tous les administrateurs, chefs de service et infirmières en chef de soutenir la campagne. Par la suite de nombreuses actions et campagnes de promotion de l'utilisation des SHA ont été déployées.

La 3ème étape a été la mise en place en 2006 d'une stratégie spécifique («mesures renforcées ») contre la diffusion des bactéries hautement résistantes émergentes (EPC, ERG) en réponse à une augmentation du nombre de cas à l'AP-HP en 2004/05 malgré l'application des «précautions contact». Ces mesures consistaient, dès qu'un cas index EPC ou ERG était identifié à : (a) signaler rapidement le cas à l'EOH centrale de l'AP-HP et alerter l'administration de l'hôpital, (b) arrêter les transferts des cas et des contacts (patients hospitalisés dans la même unité pendant la même période que les cas) vers d'autres unités de l'hôpital ou vers d'autres hôpitaux, (c) dépister les patients contact, (d) renforcer l'hygiène des mains avec les SHA et désinfecter l'environnement des patients avec un produit détergentdésinfectant, (e) en cas d'épidémie (au moins un cas secondaire identifié), regrouper les patients dans 3 secteurs distincts avec des personnels paramédicaux dédiés (secteur «cas», secteur «contacts», secteur «nouveaux patients» c.à.d. les patients nouvellement admis sans contact préalable avec des patients porteurs, (f) programmer le système d'information hospitalier pour identifier les cas et contacts en cas de réadmission. Ces mesures doivent être maintenues jusqu'à ce que l'épidémie soit considérée comme contrôlée, c'est-à-dire après sortie de tous les cas et après une période d'au moins trois mois sans nouveau cas. Pour stimuler les efforts des EOH et des administrations locales, l'EOH centrale a suivi le nombre de nouveaux cas, de nouveaux foyers épidémiques, les difficultés de mise en œuvre du programme et a régulièrement diffusés les résultats aux EOH locales et à l'administration centrale. L'EOH centrale a visité régulièrement les hôpitaux pour aider les équipes locales à appliquer le programme.

La 4ème étape a introduit en 2008 la recommandation d'identifier et dépister tout patient rapatrié d'un hôpital étranger ou ayant été récemment hospitalisé à l'étranger. Le système d'information a été programmé pour faciliter le repérage de ces patients à l'admission.

La 5ème étape a consisté en une évaluation de la gestion des excrétas dans les hôpitaux de l'AP-HP et à la diffusion de recommandations à la suite de cette évaluation. En effet, la gestion des excrétas est un sujet souvent négligé, ce qui favorise la transmission croisée des BMR digestives (EBLSE, EPC, ERG)., une enquête menée en 2012 a évalué les équipements et les pratiques des professionnels de santé pour la gestion des excréta dans 536 unités des 
soins de l'AP-HP (12) ce qui a amené à émettre des recommandations : tenue appropriée, utilisation de sacs à usage unique pour le recueil des excréta, suppression des douchettes rince-bassins, maintenance régulière des lave-bassins. Un programme de formation du personnel a été mis en place. L'évaluation de la mise en œuvre de ces recommandations a été incluse dans les indicateurs de qualité institutionnels des hôpitaux de l'AP-HP.

\section{Impact des programmes de contrôle des bactéries multirésistantes MR à l'AP-HP}

\section{Diminution de la prévalence et de l'incidence des SARM}

Entre 1993 et 2016, la proportion de SARM au sein des infections à S. aureus a diminué de $39,4 \%$ à $9,6 \%$ dans les hôpitaux de court séjour et le taux d'incidence de 1,16 à 0,33 pour 1 000 jours d'hospitalisation (figure 1) (13). La diminution de l'incidence était plus marquée en réanimation $(2,9$ à $0,5 / 1000 \mathrm{JH})$ et en chirurgie $(1,5$ à 0,4$)$ qu'en médecine $(0,7$ à 0,2$)$ et dans les établissements de réadaptation et de soins de longue durée $(0,5$ à 0,15$)$. Fait notable, la diminution a été plus marquée après le lancement de la campagne sur les solutions hydroalcooliques en 2001. Il faut noter que la prévalence des SARM a diminué fortement dans d'autres pays européens qui ont mené une politique volontariste, en particulier au Royaume Uni et en Belgique, alors qu'elle diminuait très peu dans d'autres pays, pas du tout dans d'autres et même augmentait dans d'autres (http://www.ecdc.europa.eu).

Figure 1: Évolution 1993-2016 des SARM dans les hôpitaux de l'Assistance Publique Hôpitaux de Paris : \% SARM chez S. aureus (triangle orange), taux de SARM pour 1000 jours d'hospitalisation (losanges bleu) et taux de SARM pour 100 admissions (carrés violet). Noter la cassure des courbes à partir de 2001, année du début de la campagne de promotion des solutions hydro-alcooliques.

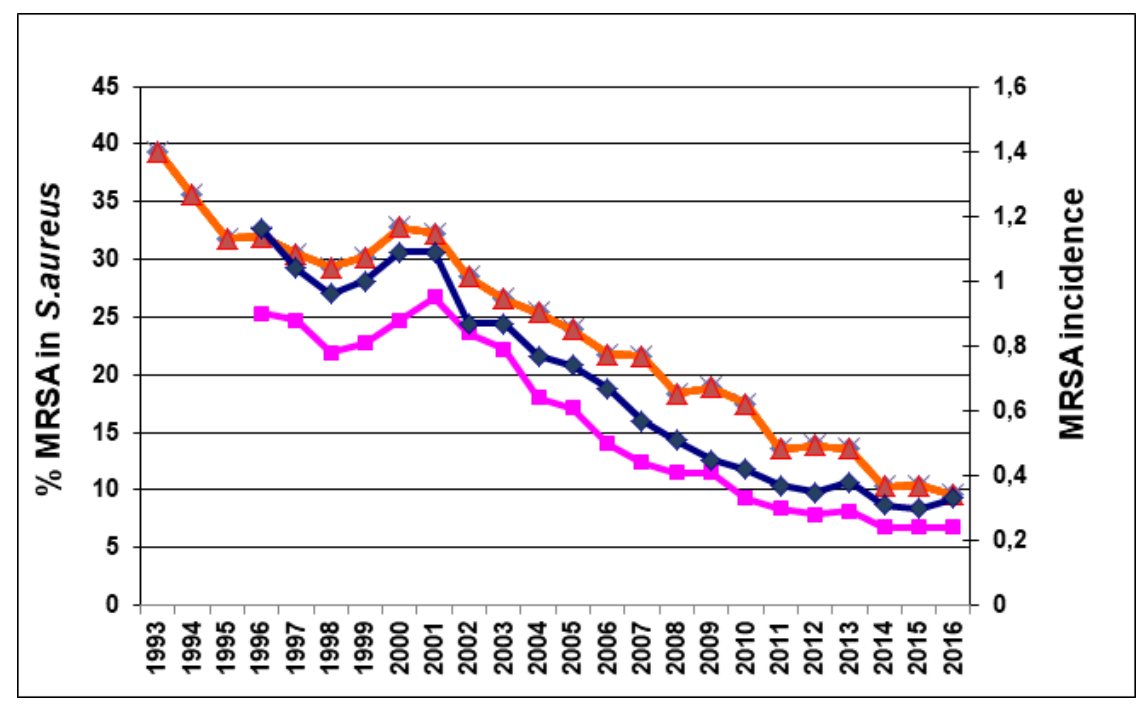

\section{Augmentation de l'utilisation des solutions hydro-alcooliques}

Les solutions hydro-alcooliques (SHA) sont des outils indispensables pour l'hygiène des mains en milieu hospitalier. À la suite des campagnes lancées en 2001, la consommation de SHA a progressivement augmenté à l'AP-HP, passant de $2 \mathrm{ml}$ par jour d'hospitalisation à 44 $\mathrm{ml}$ en 2017 (figure 2).

Figure 2: Evolution 1997-2017 de la consommation de solutions hydro-alcooliques (en ml par jour d'hospitalisation dans les hôpitaux de l'Assistance Publique - Hôpitaux de Paris. 
Noter le pic transitoire de la consommation en 2009 qui correspond à l'épidémie de grippe H1N1.

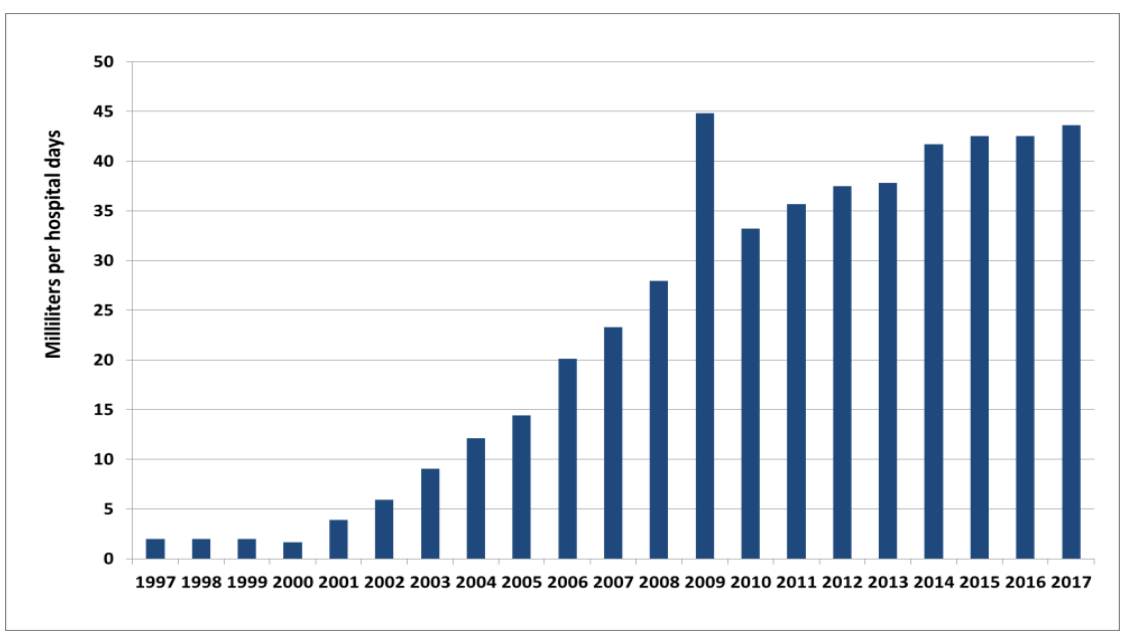

Contrôle des épidémies d'entérocoques résistants aux glycopeptides (ERG)

Le nombre moyen de cas épidémiques d'ERG a augmenté de 0,8 cas par mois (CI 95\%: 0,3 à $1,3, p=0,001)$ en 2004 et 2005 malgré les mesures «précautions contacts » qui avaient été utilisées avec succès pour contrôler la transmission croisée des SARM, mais a nettement diminué lorsque les «mesures renforcées » (voir plus haut «Programmes institutionnels » pour le détail de ces mesures) ont été mises en œuvre : diminution de 0,7 cas par mois (figure 3) (14). En clair, le nombre d'épidémies, ainsi que le nombre de cas par épidémie, était significativement plus bas après la mise en œuvre du programme.

Figure 3: Évolution 2004-2010 du nombre mensuel de cas épidémiques d'entérocoques résistants aux glycopeptides (ERG) dans les hôpitaux de l'Assistance Publique - Hôpitaux de Paris. Durant la période 2004-2005 (augmentation des ERG), les «précautions contact» ont été appliquées et les "mesures renforcées" ont été mises en place à partir de 2006 (diminution des ERG).

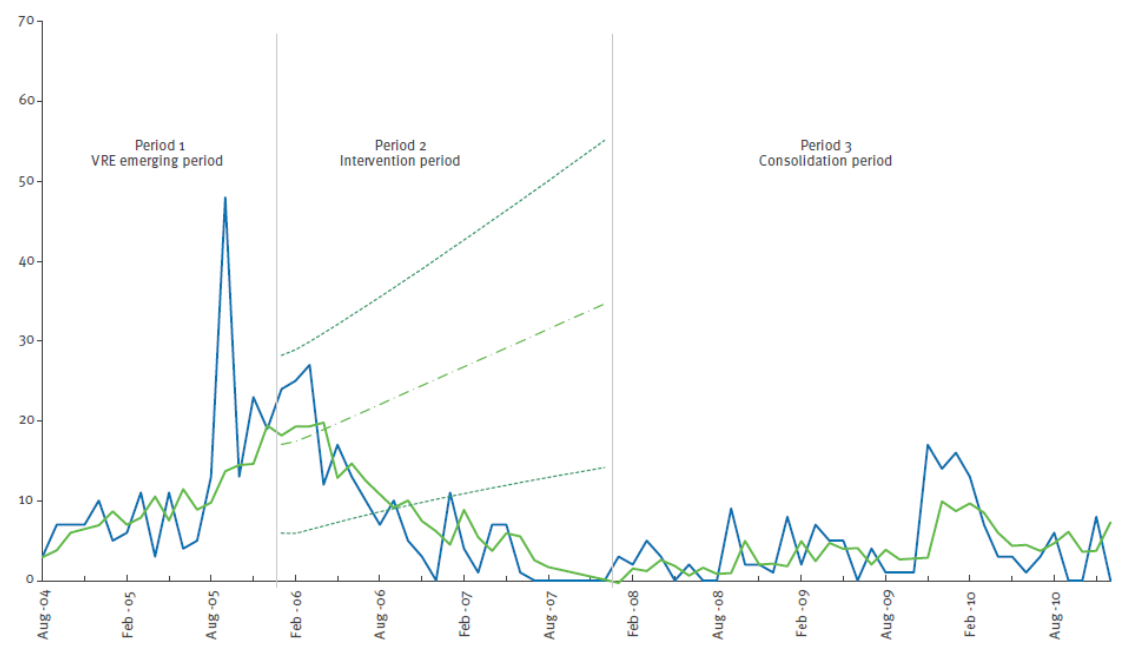

Contrôle des épidémies d'entérobactéries productrices de carbapénèmase (EPC) 
De 2004 à 2017, le nombre de cas index d'EPC est passé de moins de 10 par an jusqu'en 2009 à près de 400 en 2017 (figure 4). Cependant, malgré cette augmentation, principalement due $(\sim 70 \%)$ aux patients ayant des antécédents connus d'hospitalisation ou de séjour à l'étranger au cours de l'année précédent leur hospitalisation, la proportion de cas index ayant conduit à des cas secondaires (c.à.d. des épidémies) a diminué de 50 à 8\% (15-16) grâce aux mesures renforcées introduites en 2006 (voir plus haut «Programmes institutionnels » pour le détail de ces mesures). En effet, ici comme pour les ERG ci-dessus, le contrôle n'avait pas été obtenu par les mesures urilisées avec succès pour les SARM.

Figure 4: Évolution du nombre de cas index d'EPC (colonnes bleues) et proportion de cas index ayant conduit à des cas secondaires (épidémies) (courbe orange), dans les hôpitaux de l'Assistance Publique - Hôpitaux de Paris, 2004- 2012.

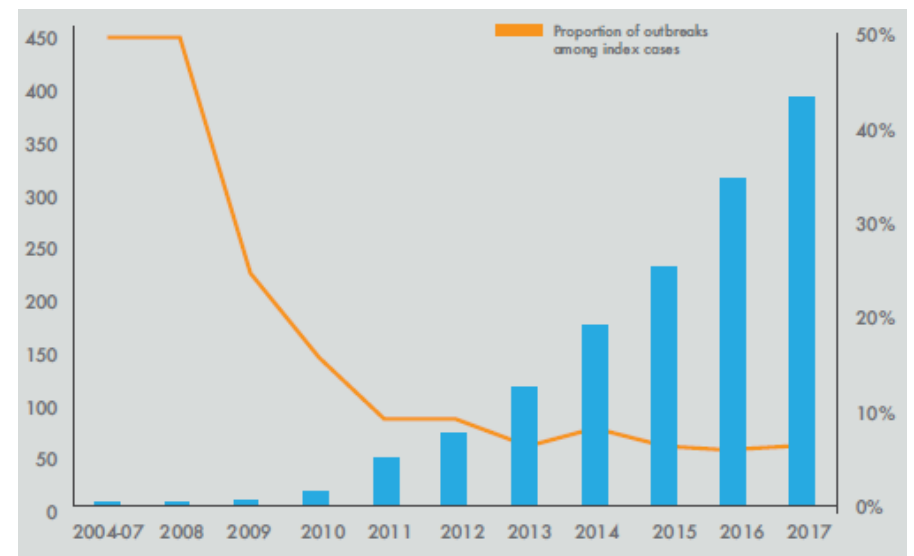

Il est important de noter que le type de mesures mises en place autour du cas index dans les 48 heures suivant son admission était crucial (16) : la proportion de cas secondaires était la plus faible en cas de personnel paramédical dédié comparativement aux «précautions contact » (procédures SARM) et aux « précautions standard » (c.à.d. hygiène « de base ») (figure 5).

Figure 5: proportion de cas secondaires (en orange) parmi les cas d'EPC, selon les mesures mises en œuvre dans les deux premiers jours autour des cas index (en gris) dans les hôpitaux de l'Assistance Publique -Hôpitaux de Paris, période 2010-2017.

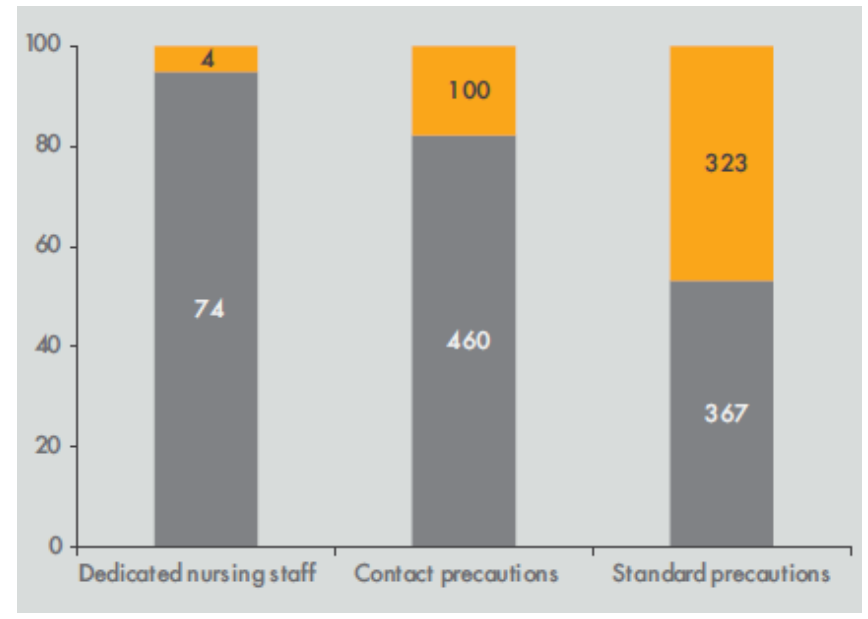

Extension des programmes de contrôle des bactéries multirésistantes au niveau national 
Les programmes initiés à l'APHP ont été étendus par les autorités sanitaires au niveau national en 1999 pour les SARM (17) et en 2013 pour les bactéries hautement résistantes émergentes (EPC, ERG) (18). Comme le montrent les statistiques de résistance produites par le système européen de surveillance Ears-net piloté par l'ECDC à Stockholm (19), surveillance qui se concentre sur les infections généralisées (bactériémies), la généralisation de ces programmes a eu des impacts très positifs en France. La proportion de SARM dans les infections à staphylocoques dorés a diminué de 33 à 13\% entre 2001 et 2017 en France alors qu'elle restait stable en Espagne, Grèce, Roumanie et Pologne et augmentait au Portugal, Hongrie et république Tchèque. La proportion d'entérobactéries résistantes aux carbapénèmes, notamment chez Klebsiella pneumoniae, espèce la plus exposée, est restée inférieures à $1 \%$ en France mais était en 2017 de 5 à 10\% au Portugal, en Pologne et Bulgarie, de 20 à 30\% en Italie et Roumanie est de 65\% en Grèce (figure 6). Enfin, la proportion d'entérocoques (espèce $E$. faecium) résistantes aux glycopeptides est restée inférieures à $1 \%$ en France mais était en 2017 de 25 à 30\% au Royaume uni, en Irlande, Pologne, Roumanie, Hongrie et Grèce (figure 6).

Figure 6: proportion de souches multirésistantes dans les bactériémies en Europe en 2017 : à gauche résistance aux carbapénèmes chez Klebsiella pneumoniae, à droite résistance aux glycopeptides chez Enterococcus faecium. Bleu clair <1\%, bleu foncé 1-5\%, jaune5-10\%, orange $10-25 \%$, rouge $25-50 \%$, pourpre $>50 \%$ (donnée Ears-net).

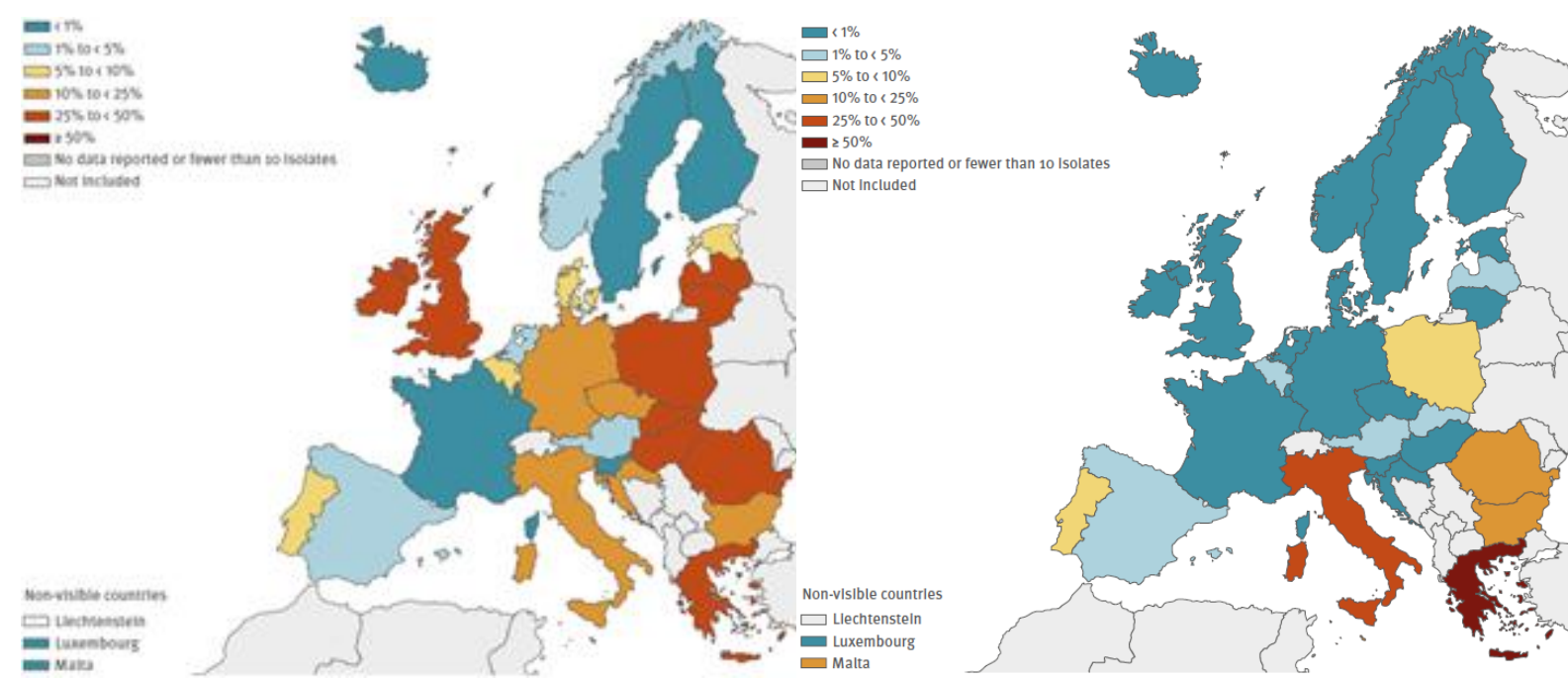

\section{Discussion}

Les programmes de contrôle de la diffusion épidémique des bactéries multirésistantes dans les hôpitaux de l'AP-HP, puis dans l'ensemble des hôpitaux de France, ont progressivement inclus des mesures ciblant (a) les SARM («précautions contact»), BMR considérée en 1993 comme prioritaires du fait d'une incidence nettement supérieure en France que dans de nombreux pays européens (b), depuis 2006, des «mesures renforcées» visant à contrôler la propagation des bactéries hautement résistantes émergentes (EPC, ERG). Parallèlement, des campagnes ont été menées pour promouvoir l'utilisation des solutions hydro-alcooliques (SHA) qui simplifie l'hygiène des mains. L'ensemble de ces mesures, comparables à celles largement décrites dans la littérature (7-11), ont permis d'entrainer une forte diminution des SARM et à contenir, du moins jusqu'à aujourd'hui, la dissémination des EPC et ERG. 
La forte augmentation à partir de 2001 de l'utilisation des SHA, qui sont les outils les plus efficaces pour l'hygiène des mains, a sans aucun doute beaucoup contribué à améliorer le niveau général d'hygiène dans les hôpitaux. La consommation de SHA est d'ailleurs utilisée comme indicateur de qualité dans les hôpitaux au niveau national ((«tableau de bord ministériel ») et européen (20), au même titre que la consommation des antibiotiques, ce qui constitue une incitation efficace. La gestion des excréta (selles et urines) est un autre point d'importance majeure pour contrôler la propagation des bactéries résistantes fécales.

Nous avons noté dans la section «Impact des programmes de contrôle » que les mesures utilisées avec succès pour contrôler la transmission croisée des SARM ("précautions contact") n'avaient pas été assez suffisantes pour contrôler les épidémies d'EPC/ERG. En effet, seules les «mesures renforcées» (arrêt des transferts des cas et des patients contact ; regroupement en secteurs distincts des cas, des patients contacts et des nouveaux patients; personnel paramédical dédié pour chaque secteur...) mises en œuvre depuis 2006 ont finalement permis un tel contrôle. Les raisons de ce fait apparemment intriguant sont en réalité faciles à expliquer. Les EPC/ERG partagent plusieurs caractéristiques critiques concernant leur potentiel de dissémination: (a) ce sont des hôtes du tube digestif et sont par conséquent facilement disséminés par voie fécale (classique voie «fécale-orale ») tandis que les SARM sont des hôtes du naso-pharynx, site bien moins propice à la dissémination, (b) leurs caractères de résistance sont portés par des éléments génétiques mobiles, ce qui augmente considérablement le risque de leur dissémination entre bactéries alors que la résistance à la méticilline du SARM est chromosomique et donc non transférable, (c) les charges bactériennes sont beaucoup plus élevées pour les EPC/ERG (jusqu'à $10^{8} /$ gr de fèces, c'est-àdire $\sim 10^{10}$ excrétés par jour par un porteur) que pour les SARM (maximum $\sim 10^{8}$ bactéries dans le nez). C'est un bon exemple de la nécessité d'adapter la politique de contrôle des infections aux caractéristiques du micro-organisme ciblé.

Le contrôle d'autres types de bactéries multirésistantes qui posent aussi des problèmes thérapeutiques nécessiterait des programmes beaucoup plus ambitieux . C'est en particulier le cas des entérobactéries résistantes aux nouvelles céphalosporines par production de $\beta$ lactamases à spectre étendu (EBLSE) qui se sont déjà répandus dans le monde entier dans les hôpitaux, mais aussi dans la communauté, le milieu animal et l'environnement, . Pour ce type de bactéries à transmission fécale-orale, les programmes devraient inclure l'amélioration de l'hygiène dans la population générale (hygiène fécale dans les écoles et autres communautés fermées, hygiène au sein de la famille ...), des politiques environnementales fortes (traitement des eaux usées et utilisation des sous-produits de ce traitement, contrôle des aliments ...), ainsi que l'organisation de l'élevage, afin de réduire les chaînes complexes de transmission. Le rapport Carlet mentionné plus haut souligne bien le rôle important joué par environnement dans la dissémination des bactéries résistantes, et de leurs gènes de résistance, qui, déversées dans l'environnement par les activités humaines (notamment via les eaux usées des hôpitaux, des collectivités, des élevages...), polluent les rivières et les sols et interagissent avec leur milieu. Si nous échouons à mettre en place de tels programmes intégrés, l'utilisation des antibiotiques efficaces pour traiter les infections à EBLSE, en particulier les carbapénèmes, augmentera, ce qui favorisera l'émergence des EPC, ultime étape de la multi-résistance chez les bacilles à Gram négatif.

Au total, la longue expérience (plus de 20 ans) des programmes de contrôle de la résistance aux antibiotiques dans les hôpitaux français et exposée dans le présent chapitre, montre que la dissémination par transmission croisée des bactéries multirésistantes peut être fortement limitée même à l'échelle de grandes institutions hospitalières, à condition que tous les acteurs, 
personnels médicaux et paramédicaux, hygiénistes, microbiologistes et administrateurs soient convaincus, stimulés et impliqués.

Fait important, les résultats positifs enregistrés ont été obtenus alors même que la consommation d'antibiotiques n'a pas diminué de façon notable dans nos hôpitaux durant la période considérée (la diminution observée en 2002-03 a depuis été annulée par une reprise des consommations [21]) malgré des actionsprobablement trop timides, ce qui montre bien l'importance clef des mesures d'hygiènes. Si l'on prend l'ensemble de la période 1993-2016 (celle des résultats exposés ici), la consommation moyenne des antibiotiques n'a pas diminué dans les hôpitaux de l'APHP et a fluctué autour de 520 doses définies journalières pour 1000 journées d'hospitalisation. Lorsque la consommation des antibiotiques aura nettement diminué en médecine humaine (sur la base des données comparatives européennes, on pourrait diminuer cette consommation en France par un facteur 2 à 3 sans grand risque), il persistera une forte pression de sélection liée aux traitements antibiotiques indispensables, infiniment plus importante qu'avant l'ère des antibiotiques. Ce sont les actions limitant la dissémination des bactéries résistantes qui permettront de contrebalancer les effets de cette pression de sélection résiduelle.

En conclusion, à l'instar des pollutions atmosphériques engendrées par l'Homme, l'antibiorésistance, elle aussi engendrée par l'Homme, pollue notre planète. Elle impacte l'ensemble des écosystèmes et menace la sécurité sanitaire des générations futures et doit donc être intégrée au vaste sujet du développement durable.

\section{Glossaire}

Résistance naturelle aux antibiotiques : caractéristique propre à une espèce bactérienne. Exemple : l'espèce Escherichia coli est naturellement resistante à la penicillin G, à l'érythromycine, à la vancomycine.

Résistance acquise aux antibiotiques : caractéristique propre à une souche devenue résistante à un antibiotique auquel l'espèce est naturellement sensible, suite à la modification de son génome (mutation chromosomique, acquisition de gènes étrangers à partir d'autres bactéries). Exemple : souche de Escherichia coli résistante à l'amoxicilline par production de pénicillinase plasmidique.

SARM : Staphylococcus aureus résistant à la méticilline.

EBLSE : entérobactéries (Escherichia coli, Klebsiella pneumoniae...) résistante aux céphalosporines de 3ème generation (cefotaxime, ceftriaxone) par production de $\beta$-lactamases à spectre étendu (BLSE).

EPC : entérobactéries (Escherichia coli, Klebsiella pneumoniae...) résistante aux carbapenems par production de carbapénèmases

ERG : entérocoque (Enterococcus faecium...) résistant aux glycopeptides.

Pression de selection antibiotique : contrainte exercée par l'utilisation des antibiotiques en santé humaine et animale, qui entraîne la sélection des bactéries les plus résistantes (évolution darwinienne).

Transmission croisée des bactéries résistantes : dissémination des bactéries résistantes sélectionnées par la pression de sélection, directement par transmission au sein des populations humaines et animales («transmission croisée »), ou indirectement via l'environnement. 


\section{Références}

1. Organisation Mondiale de la Santé (OMS), 2016, Plan d'action mondial pour combattre la Résistance aux Antimicrobiens, disponible par http://apps.who.int/iris/bitstream/handle/10665/249548/9789242509762fre.pdf;jsessionid=0621CEC5A57AD135A25613B2C17B2F35? sequence $=1$

2. Organisation des Nations Unies (ONU), 21 juin 2016, High-level Meeting on Antimicrobial Resistance, disponible parhttps://www.un.org/pga/71/event-latest/highlevel-meeting-on-antimicrobial-resistance/

3. Commission européenne, 15 novembre 2011, Action Plan against the rising threats from Antimicrobia, disponible parl Resistance. http://ec.europa.eu/health/amr/sites/amr/files/communication_amr_2011_748_en.pdf

4. Commission européenne, 29 juin 2017, A European Action Plan against Antimicrobial Resistance, disponible par

https://ec.europa.eu/health/amr/sites/amr/files/amr_action_plan_2017_en.pdf

5. Rapport Carlet et Le Coz, juin 2015, Tous ensemble, sauvons les antibiotiques, disponible parhttp://solidarites-sante.gouv.fr/IMG/pdf/rapport_antibiotiques.pdf

6. Colomb-Cotinat M, Lacoste J, Brun-Buisson C, Jarlier V, Coignard B, Vaux S. Estimating the morbidity and mortality associated with infections due to multidrugresistant bacteria (MDRB), France, 2012. Antimicrob Resist Infect Control, 2016, 5:56

7. Wenzel RP, Reagan DR, Bertino JS Jr, Baron EJ, Arias K. Methicillin-resistant Staphylococcus aureus outbreak: a consensus panel's definition and management guidelines. Am J Infect Control. 1998 Apr;26(2):102-10.

8. Report of a combined Working Party of The Hospital Infection Society and The British Society for Antimicrobial Chemotherapy. Revised guidelines for the control of epidemic methicillin-resistant Staphylococcus aureus. Hosp Infect 1990; 16: 351-377.

9. Ridwan B, Mascini E, van Der Reijden N, Verhoef J, Bonten M. What action should be taken to prevent spread of vancomycin resistant enterococci in European hospitals? BMJ. 2002;324(7338):666-8.

10. Schwaber MJ, Lev B, Israeli A, Solter E, Smollan G, Rubinovitch B, et al. Containment of a country-wide outbreak of carbapenem-resistant Klebsiella pneumoniae in Israeli hospitals via a nationally implemented intervention. Clin Infect Dis. 2011;52(7):848-55.

11. Kochar S, Sheard T, Sharma R, Hui A, Tolentino E, Allen G, et al. Success of an infection control program to reduce the spread of carbapenem-resistant Klebsiella pneumoniae. Infect Control Hosp Epidemiol. 2009;30(5):447-52.

12. Lepainteur M, Nérome S, Bendjelloul G, Monteil C, Cottard-Boulle B, Nion-Huang M, Jarlier V, Fournier S; Network of Infection Control Teams of Assistance Publique Hôpitaux de Paris. Evaluation of excreta management in a large French multi-hospital institution. J Hosp Infect 2015;91:346-50.

13. Jarlier V, Trystram D, Brun-Buisson C, Fournier S, Carbonne A, Marty L, et al. Curbing methicillin-resistant Staphylococcus aureus in 38 French hospitals through a 15-year institutional control program. Arch Intern Med 2010;170:552-9 
14. Fournier S, Brossier F, Fortineau N, Gillaizeau F, Akpabie A, Aubry A, et al. Long-term control of vancomycin-resistant Enterococcus faecium at the scale of a large multihospital institution: a seven-year experience. Euro Surveill. 2012;17(30)

15. Fournier S, Monteil C, Lepainteur M, Richard C, Brun-Buisson C, Jarlier V, et al. Longterm control of carbapenemase-producing Enterobacteriaceae at the scale of a large French multihospital institution: a nine-year experience, France, 2004 to 2012. Euro Surveill 2014;19(19)

16. Fournier S, Desenfant L, Monteil C, Nion-Huang M, Richard C, Jarlier V; Efficiency of different control measures for preventing carbapenemase-producing enterobacteria and glycopeptide-resistant Enterococcus faecium outbreaks: a 6-year prospective study in a French multihospital institution, January 2010 to December 2015. Euro Surveill. 2018;23(8).

17. Maitrise de la diffusion des bactéries multirésistantes aux antibiotiques.

Recommandations pour les établissements de santé ; Direction générale de la Santé 1999

18. Prévention de la transmission croisée des Bactéries Hautement Résistantes aux antibiotiques émergentes (BHRe), Haut Conseil de la santé publique, Juillet 2013

19. Statistiques de résistance aux antibiotiques dans les bactériémies en Europe, disponible par http://www.ecdc.europa.eu/en/publications

20. Point prevalence survey of healthcare-associated infections and antimicrobial use in European acute care hospitals 2011-2012, disponible par www.ecdc.europa.eu

21. La consommation ; d'antibiotiques en France en 2016, Agence nationale de sécurité du médicament et des produits de santé (ANSM), Décembre 2017 